\title{
ON THE BIRTH OF THE NEVANLINNA THEORY
}

\section{OLLI LEHTO}

Classical value distribution theory deals with the study of the density of the points in the plane at which an analytic function takes a prescribed value. Polynomials, for which complete results can at once be obtained, served as a model when the study of entire functions was started about a hundred years ago. By the turn of the century, Borel had succeeded in combining and improving results of Picard, Poincaré and Hadamard in such a way that a value distribution theory began to take shape. Further progress was made during the first two decades of this century by a greatly increased number of mathematicians, albeit without any particularly striking results. The theory of meromorphic functions resisted such a development: apart from a few exceptions, it was difficult even to formulate the problems studied in connection with entire functions.

In the early twenties the state of affairs underwent a discontinuous change due to Rolf Nevanlinna. He succeeded in creating a far-reaching value distribution theory for meromorphic functions, in such a way that it contained as a special case the theory of entire functions in an improved form. The Nevanlinna theory came into being through the work he did in the years 1922-24. However, the impact of the new theory was so profound that the process of birth persisted for a period of about ten years.

Our description of this period will be preceded by a brief summary of the anteNevanlinna value distribution theory.

\section{Value distribution theory before Nevanlinna}

1.1. Rational functions. The value distribution of a polynomial is very symmetric, and there is a simple relation between the number of its zeros and its growth near infinity. A polynomial $f$ of degree $n \geqq 1$ has $n$ zeros if each zero is counted according to its multiplicity. Since $f+$ constant also is a polynomial of degree $n$, it follows that $f$ takes every complex value precisely $n$ times. With the aid of its zeros $a_{1}, a_{2}, \ldots, a_{n}$, the function $f$ can be represented in the form

$$
f(z)=c\left(z-a_{1}\right)\left(z-a_{2}\right) \ldots\left(z-a_{n}\right) .
$$


The degree $n$ also tells exactly how rapidly $|f(z)|$ grows as $z \rightarrow \infty$ : we have the asymptotic equation

$$
\lim _{z \rightarrow \infty} f(z) / z^{n}=c \quad(\neq 0, \infty) .
$$

If an arbitrary non-constant rational function $f$ is regarded as a self-mapping of the extended plane, then again $f$ takes every value the same finite number of times. An analogue of (1) is obtained, since $f$ is a quotient of two polynomials, whereas there is no counterpart for the growth condition (2).

These properties of polynomials and general rational functions find their analogues among entire and meromorphic functions. This fact unfolded gradually in the course of a long development which culminated in the work of Nevanlinna.

1.2. Weierstrass product representation. The study of the zeros of entire functions began about a century ago. In 1876, Weierstrass [34] proved that to every sequence $\left(a_{n}\right)$ of complex numbers with $\left|a_{1}\right| \leqq\left|a_{2}\right| \leqq \ldots, \lim a_{n}=\infty$, there corresponds an entire function having these $a_{n}$ as zeros. The proof was by direct construction: a solution is provided by a canonical product

where

$$
\prod_{n=1}^{\infty} E\left(z / a_{n} ; k_{n}\right)
$$

$$
E(w ; k)=(1-w) \exp \left(w+w^{2} / 2+\ldots+w^{k} / k\right) .
$$

(If $a_{n}=0$ is a zero, one writes $z$ instead of $1-z / a_{n}$ in (3).) The non-negative integers $k_{n}$ are chosen so as to make the product (3) convergent. If there is a $\lambda>0$ such that $\sum\left|a_{n}\right|^{-\lambda}$ converges, then a fixed $k$, the largest integer $<\lambda$, will do for that purpose.

All entire functions having the given $a_{n}$ as zeros are obtained from the Weierstrass product formula

$$
f(z)=e^{g(z)} \prod_{n=1}^{\infty} E\left(z / a_{n} ; k_{n}\right),
$$

where $g$ is an arbitrary entire function. This is the counterpart of the polynomial formula (1).

Formula (4) was the starting point for the attempts to generalize from polynomials to entire functions the result on the relation between the number of zeros and the growth as $z \rightarrow \infty$. There is of course the striking difference that while the zeros determine a polynomial up to a multiplicative constant, they determine an entire function only up to a zero-free function $e^{g}$. Since $e^{g}$ can be of arbitrarily rapid growth, there cannot be a full counterpart to the asymptotic equation (2).

1.3. Genus of entire functions. Soon after Weierstrass had discovered formula (4), Laguerre noticed the need to classify entire functions using a notion which in some sense assumes the role of the degree of polynomials. Suppose an entire 
function $f$ has so few zeros $a_{n}$ that the series $\sum\left|a_{n}\right|^{-\lambda}$ converges for some $\lambda>0$. Then there is a smallest integer $p \geqq 0$ such that $\sum\left|a_{n}\right|^{-(p+1)}$ is convergent, and the canonical product (3) associated with $f$ converges for $k_{n}=p$. Let us assume, furthermore, that in the Weierstrass representation

$$
f(z)=e^{g(z)} \prod E\left(z / a_{n} ; p\right)
$$

the function $g$ is a polynomial of degree $q \geqq 0$. Then $f$ is said to be of genus $k=\max (p, q)$. If $f$ has so many zeros that no $\lambda$ of the above kind exists or if such a $\lambda$ exists but $g$ in (5) is not a polynomial, then $f$ is said to be of infinite genus.

Let

$$
M(r)=\max _{|z| \leqq r}|f(z)|
$$

denote the maximum modulus of the entire function $f$. An analogue of (2) would be a bound for $M(r)$ in terms of the number of zeros of $f$. When such a bound was being sought in the 1880's, the very natural restriction was imposed on $f$ that $f$ be of finite genus. This assumption not only implies a bound for the number of zeros of $f$ but it also keeps the non-zero term $e^{g}$ under control.

In 1883 Poincaré [29] proved results solving this problem: An entire function of genus $k$ satisfies the inequality

$$
\log M(r)=o\left(r^{k+1}\right)
$$

as $r \rightarrow \infty$.

1.4. Order of entire functions. The converse problem to Poincaré's theorem is to derive an upper bound for the number of zeros $a_{n}$ of an entire function $f$ when a bound for its maximum modulus is given. Here no additional a priori assumptions about $f$ need be made.

A bound for the maximum modulus can appropriately be given by aid of the number $\varrho=\inf \left\{\lambda \mid \log M(r)=O\left(r^{\lambda}\right)\right\}$, which is also obtained as the upper limit

$$
\varrho=\limsup _{r \rightarrow \infty} \frac{\log \log M(r)}{\log r} .
$$

This is called the order of the function. Its formal definition was given in 1897 by Borel [3] who realized its central role in the theory of entire functions.

Before that Hadamard [9] had proved a number of results which yield a solution to the converse problem: If an entire function $f$ is of finite order $\varrho$, then the series $\sum\left|a_{n}\right|^{-(\varrho+\varepsilon)}$ converges for every $\varepsilon>0$. It follows that the genus of $f$ is finite, and if $k<\varrho<k+1$, where $k$ is an integer, then $f$ is of genus $k$.

Hadamard's theorem can be stated in a more general form which at the same time better illustrates the meaning of the convergence of the series. For this purpose, let us consider an increasing sequence $\left(r_{n}\right)$ of positive numbers wiht $\lim r_{n}=\infty$, and call the infimum of the numbers $\lambda$ for which $\sum r_{n}^{-\lambda}$ converges the convergence exponent of $\left(r_{n}\right)$. 
Let $n(r, a)$ denote the number of $a$-points of an entire function $f$ in the disc $|z| \leqq r$, i.e. the number of the roots $z_{i}$ of the equation $f(z)=a$, each root being counted according to its multiplicity. A simple calculation shows that the integral $\int^{\infty} n(t, a) t^{-(\lambda+1)} d t$ and the series $\sum\left|z_{i}\right|^{-\lambda}$ are simultaneously convergent. It follows that the convergence exponent $\alpha$ of the sequence $\left(\left|z_{i}\right|\right)$ admits the characterization

$$
\alpha=\limsup _{r \rightarrow \infty} \frac{\log n(r, a)}{\log r} .
$$

Let us now return to Hadamard's theorem. Since $f$ and $f-a$ are of the same growth, we can replace the zeros of $f$ by any of its $a$-points. Therefore, in view of the characterization (7), Hadamard's theorem allows the following reformulation: For an entire function of order $\varrho$, the a-points are so distributed that

for every value of a.

$$
\limsup _{r \rightarrow \infty} \frac{\log n(r, a)}{\log r} \leqq \varrho
$$

1.5. Theorems of Picard and Borel. As early as 1879, Picard [29] had proved the famous theorem that a non-constant entire function can omit only one value. The very short proof was based on the use of the elliptic modular function and did not utilize the basic representation formula (4). For quite a while this result remained rather isolated, until Borel [3] in 1897 proved a part of it in a much sharper form. This connected Picard's theorem with the then existing theory about the distribution of values of entire functions.

In inequality (8) the left side can of course be strictly less than $\varrho$, since $n(r, a)$ can even be zero for every $r$. On the other hand, Picard's theorem says that $n(r, a)$ can vanish identically for one value $a$ only. Borel's theorem goes much farther; in above notation it can be expressed as follows: For an entire function of finite order $\varrho$, equality holds in (8), with the possible exception of one single value a.

Thus Borel's theorem solved at the same time the two problems about the relations between $M(r)$ and $n(r, a)$ treated in Poincaré's and Hadamard's theorems; for Poincaré's theorem the more general viewpoint of considering $a$-points rather than zeros is essential. Borel's theorem shows that not only polynomials but all non-constant entire functions of finite order exhibit a remarkable symmetry in the distribution of their values and that, up to one value, the number of $a$-points is determined by the rate of growth of the function.

Borel also gave indications of how to extend the theorem to functions of infinite order. This was done by Blumenthal [2] in 1910.

1.6. Repercussions of Borel's theorem. Borel's work seems to have increased considerably the interest in entire functions. His results led to a number of new problems of which some were explicitly posed by Borel himself, such as how to 
improve his theorem by the introduction of measures of growth finer than the order. Also, Borel's method, which was based on estimates on the minimum modulus of an entire function and on the growth of its derivative, gave rise to many questions not directly connected with the relations between the maximum modulus and the function $n(r, a)$. It is hardly an exaggeration to say that during the first two decades of our century, Borel's theorem dominated the theory of entire functions. Valiron, one of the most active and successful of Borel's successors, said in his lecture [32] in the 1920 International Congress of Mathematicians in Strasbourg: "C'est à cette proposition que se rattachent plus ou moins directement tous les travaux ultérieurs sur les fonctions entières."

Having been prior to that time almost exclusively in the hands of French mathematicians, research on entire functions started to spread to other countries following the turn of the century. One of Borel's first successors was Ernst Lindelöf, Nevanlinna's future teacher and his father's cousin. Lindelöf's paper [12] of 1902 contains, among other things, simplified proofs of the main results of the Poincaré-Hadamard-Borel theory. In reviewing this theory in the monograph [5], Borel adopted Lindelöf's respresentation: "M. Lindelöf a retrouvé, par une voie plus rapide, la plupart de ces résultats."

The second edition of Borel's monograph [4] "Leçons sur les fonctions entières", which appeared in 1921, included developments subsequent to Borel's theorem. In the preface Borel motivated the new printing by the remark that the fundamentals of the theory of entire functions had not changed since the appearance of the first edition which was based on lectures given in 1897-98. An updated theory was presented in the supplementary chapter "Progrès de la théorie des fonctions entières depuis 1900" written by Valiron. This chapter also contained a bibliography of almost a hundred entries covering the period 1900-20.

During this time the program suggested by Borel was largely carried out, by Wiman, Lindelöf, Valiron, and others. Borel's theorem was refined in various ways by the use of classifications for entire functions more accurate than the one based on the notion of order. Moreover, Valiron showed that Borel's theorem remains valid for functions holomorphic in the unit disc provided that the maximum modulus $M(r)$ tends to $\infty$ sufficiently rapidly as $r \rightarrow 1$. Valiron also called special attention to Jensen's formula, already used by Jensen himself [11] and by Lindelöf [12] to study value distribution, and may have influenced Nevanlinna by the way he wrote it (cf. Sections 2.3 and 2.4).

1.7. Results on meromorphic functions. Let us consider now a function $f$ meromorphic in the complex plane. If $f$ omits a finite value $c$, then $1 /(f-c)$ is an entire function. Therefore, Picard's theorem generalizes immediately: A function meromorphic in the complex plane and omitting three values is a constant.

In contrast to this, before Nevanlinna there had been no satisfactory way to extend to meromorphic functions the Poincaré-Hadamard-Borel theory of entire 
functions. Since a meromorphic function can take the value $\infty$, the basic problem of studying the relations between the maximum modulus $M(r)$ and the counting function $n(r, a)$ becomes meaningless.

Borel [5] had made an attempt to circumvent this difficulty. For a given meromorphic $f$, form the Weierstrass canonical product $h$ of lowest possible genus using as zeros the poles of $f$. Then $f h=g$ is an entire function, and so $f$ admits a representation

$$
f=g / h
$$

as a quotient of two entire functions. Borel defined the order of $f$ to be the greater of the orders of $g$ and $h$. With this definition, Borel succeeded in generalizing his theorem: a meromorphic function $f$ of finite order $\varrho$ satisfies the inequality (8), and equality holds with the possible exception of two values of $a$.

The representation (9), on which this theorem depends, is unique only if the genus of the denominator $h$ is finite. Also, being based on (9), the required condition that $f$ be of finite order is very implicit. Later development has shown that (9) does not offer a convenient starting point for building a general theory for meromorphic functions.

\section{Nevanlinna's first main theorem}

2.1. Beginning of Nevanlinna's research. The only source today for learning about Nevanlinna's gradual penetration into the properties of meromorphic functions are his original mathematical papers. After his death quite a number of copybooks and scattered notes from the early twenties were found, but they turned out to be disappointing in that they did not shed new light on the actual birth of the Nevanlinna theory. Either they were manuscripts, virtually identical with published papers, or else lecture notes which did not reveal the ripening of Nevanlinna's ideas beyond what can be read in his printed publications.

On the other hand, Nevanlinna's carefully written papers, which came out in quick succession in 1922-25 and in which underlying ideas are often explained, make exciting reading. They show how the author step by step unveiled the mysteries surrounding meromorphic functions until, in 1925, the essentials of a theory were there to be read in a pure and elegant form.

In 1922, when Nevanlinna was 26 and three years had elapsed since the appearance of his doctoral thesis on bounded analytic functions, his interest was focused on value distribution problems. In this he was stimulated and supported by his teacher Lindelöf, then one of the world's foremost experts in the field; throughout his life, Nevanlinna held Lindelöf in the highest esteem. It is not unlikely that Lindelöf contributed to Nevanlinna's interest in the potential-theoretic method which was one key to his success. 
Clearly very important for Rolf Nevanlinna at this stage was the contact and actual joint work with his older brother Frithiof. In his Finnish memoirs "Muisteltua" ([28]) Rolf gave full credit to Frithiof: "Discussions with him have decisively contributed to the success of my work... . I do not think I have received so much significant inspiration from anyone else."

2.2. Potential-theoretic method. Nevanlinna's very first value distribution paper, a Comptes Rendus note [14], appeared in May 1922; it announced results concerning functions holomorphic in an angle. Two months later Nevanlinna gave a talk ([15]) in the Scandinavian Congress of Mathematicians in Helsinki. Its title "Über die Anwendung des Poisson'schen Integrals zur Untersuchung der Singularitäten analytischer Funktionen" indicates the method whose importance Nevanlinna repeatedly stressed, then and later.

This method was inaugurated in the comprehensive joint paper [13] with Frithiof Nevanlinna, which was communicated in November 1922. The work begins as follows: "This paper is devoted to the presentation and the most important applications of a general function-theoretic method. The essence of the method is to utilize, as completely as possible, the simple fact that the logarithm of the absolute value of a meromorphic function $f(x)$ is a single-valued harmonic function which at the zeros and poles of the function $f(x)$ becomes negatively and positively logarithmically infinite."

In reviewing [13] for "Jahrbuch über die Fortschritte der Mathematik" G. Szegö wrote: "Besonders reizvoll wirkt an diesen Untersuchungen, ausser ihrer geradezu klassischen formalen Eleganz, die Abgeschlossenheit der Resultate und die Einfachheit der befolgten Methode." Ahlfors [1] said that after the appearance of [13], function theory was no longer the same as before.

From the point of view of direct impact on the value distribution theory, [13] must still be regarded as preparatory. But the function $\log ^{+}|f|$, soon to gain great importance, was there, and the authors introduced the integral

$$
\int_{0}^{2 \pi} \log \left|f\left(r e^{i \varphi}\right)\right| d \varphi
$$

to measure the growth of $f$.

Even years later, when his theory was essentially developed, Nevanlinna emphasized the role of the method. In the opening lines of the monograph [21] "Le théorème de Picard-Borel et la théorie des fonctions méromorphes" he wrote that the theory to be developed is based on the systematic use of certain general formulas which can easily be derived by aid of Green's formulas.

2.3. The Poisson-Jensen formula. The basic formula which Nevanlinna systematically utilizes can be derived as follows. Let $f$ be meromorphic in the closure of a domain $D$ whose boundary consists of a finite number of analytic curves, with 
the zeros $a_{h}$ and poles $b_{k}$ in $D$. If $z \rightarrow g(z, \zeta)$ is the Green's function of $D$ with singularity at $\zeta$, then the function

$$
z \rightarrow \log |f(z)|+\sum g\left(z, a_{h}\right)-\sum g\left(z, b_{k}\right)
$$

is harmonic in $D$ and has the same boundary values as $\log |f|$. Application of Green's formula to (1) then gives $\log |f(z)|$ in terms of the boundary values of $\log |f|$ and of the zeros $a_{h}$ and poles $b_{k}$.

In the special case where $D$ is the disc $|z|<r$, Poisson's formula can be applied to (1). Because $g(z, \zeta)=\log \left|\left(r^{2}-z \bar{\zeta}\right) / r(z-\zeta)\right|$, the ensuing result is very explicit:

$$
\begin{aligned}
\log |f(z)|= & \frac{1}{2 \pi} \int_{0}^{2 \pi} \log \left|f\left(r e^{i \theta}\right)\right| \frac{r^{2}-|z|^{2}}{r^{2}+|z|^{2}-2 r|z| \cos (\theta-\arg z)} d \theta \\
& -\sum \log \left|\frac{r^{2}-\bar{a}_{h} z}{r\left(z-a_{h}\right)}\right|+\sum \log \left|\frac{r^{2}-\bar{b}_{k} z}{r\left(z-b_{k}\right)}\right| .
\end{aligned}
$$

The validity of (2) is clear if $f(z) \neq 0, \infty$ on the boundary $|z|=r$. But even without this restriction the integral in (2) converges and equation (2), which Nevanlinna calls the Poisson-Jensen formula, is true.

Particularly important is the special case $z=0$ :

$$
\log |f(0)|=\frac{1}{2 \pi} \int_{0}^{2 \pi} \log \left|f\left(r e^{i \theta}\right)\right| d \theta-\sum \log \frac{r}{\left|a_{h}\right|}+\sum \log \frac{r}{\left|b_{k}\right|} .
$$

In Nevanlinna's hands this simple formula, established by Jensen [11] in 1899, was to lead to amazing consequences.

2.4. Counting function and proximity function. In his thesis [31] of 1913, Valiron had used Jensen's formula (3) and taken one step towards the final form into which Nevanlinna transformed it. If again $n(r, a)$ denotes the number of $a$-points of $f$ in $|z| \leqq r$, then

$$
\sum \log \frac{r}{\left|a_{h}\right|}=\int_{0}^{r} \log \frac{r}{t} d n(t, 0)=\int_{0}^{r} \frac{n(t, 0)}{t} d t .
$$

Similarly, Valiron writes

$$
\sum \log \frac{r}{\left|b_{k}\right|}=\int_{0}^{r} \frac{n(t, \infty)}{t} d t
$$

In the monograph [33], Valiron explicitly stressed the importance of Jensen's formula and used (4) and (4').

A year after the joint work [13], Nevanlinna was ready in November 1923 to publish a large paper "Untersuchungen über den Picard'schen Satz" ([16]). It represented a real breakthrough. Nevanlinna introduced and made systematic use for the first 
time of two of his basic concepts, the counting function $N$ and the proximity function $m$, there with the notation

$$
N(r, f)=\int_{0}^{r} \frac{n(t, 0)}{t} d t, \quad m(r, f)=\frac{1}{2 \pi} \int_{0}^{2 \pi} \log \left|f\left(r e^{i \theta}\right)\right| d \theta .
$$

(If $z=0$ is a zero or a pole of $f$, then both sides of (3) become infinite. Likewise, $f(0)=0$ makes the above $N(r, f)$ infinite. This state of affairs can be readily corrected by a minor modification; in the following we shall disregard it.)

The functions $N$ and $m$ arose from a reformulation of Jensen's formula (3). In view of (4) and (4'), the two sums there are $N(r, f)$ and $N(r, 1 / f)$. But the decisive step was to use the simple identity

$$
\log x=\stackrel{+}{\log x-\log ^{+}(1 / x)}
$$

which allowed Nevanlinna to decompose the curve integral in (3) into $m(r, f)$ $m(r, 1 / f)$. Thus he rewrote (3) in the form

$$
m(r, f)+N(r, 1 / f)=m(r, 1 / f)+N(r, f)+\log |f(0)| .
$$

Ahlfors [1] said that this was the moment when the Nevanlinna theory of meromorphic functions was born.

In retrospect, the use of (6) seems very simple. But it was scarcely accidental: already in the previous work [13] Nevanlinna had noticed the importance of the proximity function $m$.

We shall come back to paper [16] in Section 3.1, having first discussed direct consequences of formula (7).

2.5. Characteristic function. Nevanlinna required a few more months to realize the full meaning of the equation (7). In January 1924 he published a Comptes Rendus note [17] which contained several new notions and results. Changing the notation a little he now put

$$
N(r, a)=\int_{0}^{r} \frac{n(t, a)}{t} d t, \quad m(r, a)=\frac{1}{2 \pi} \int_{0}^{2 \pi} \log \frac{1}{\left|f\left(r e^{i \theta}\right)-a\right|} d \theta .
$$

As the first trace of his characteristic function, he wrote $T(r, a)=m(r, a)+N(r, a)$; formula (7) then assumes the very simple form $T(r, \infty)=T(r, 0)+\log |f(0)| . \mathrm{He}$ proved, as the first step towards the invariance of $T(r, a)$ with respect to $a$, that for any two values $a$ and $b$, the ratio $T(r, a) / T(r, b)$ tends to 1 as $r \rightarrow \infty$.

Finally, in the Comptes Rendus note [19] of July 1924, Nevanlinna drew the final conclusion from (7). In many ways this is a central result in the value distribution theory; in [20] and later, Nevanlinna called it the First Main Theorem: 
To each function non-constant and meromorphic in $|z|<R \leqq \infty$, there corresponds a continuous increasing function $T(r)$ such that for every value $a$,

$$
T(r)=m(r, a)+N(r, a)+O(1),
$$

where $O$ (1) remains bounded as $r \rightarrow R$.

Formula (9) follows readily from (7) if the notation in (8) is used; one can take, for instance, $T(r)=m(r, \infty)+N(r, \infty)$.

The function $T$, the characteristic of $f$, was to play a decisive role in the theory of meromorphic functions. In the monograph [10] W. K. Hayman, one of the leading contemporary experts on the Nevanlinna theory, wrote about it: "In the course of the book it is hoped to show the tremendous gain in elegance, clarity, and depth which results from this basic concept, even in the special case of entire functions."

Suppose that $T(r) \rightarrow \infty$ as $r \rightarrow R$; if $R=\infty$, this is always the case. The first main theorem then says that if $f$ takes a value $a$ fewer than average, i.e. if $N(r, a)$ is relatively small, then there is always a compensation: the function $f$ approximates such a value stronger than average, i.e. the proximity function $m(r, a)$ is relatively large. To quote Nevanlinna, the total affinity of $f$ towards each value $a$ is the same, independent of $a$. In this sense, the value distribution of meromorphic functions is analogous to that of rational functions.

In addition to its intrinsic interest, the first main theorem (9) proved to be an important technical tool for handling meromorphic functions.

\section{Nevanlinna's second main theorem}

3.1. Second main theorem for three values. The relation (9) of the first main theorem gave rise to the question of the relative size of the components $m(r, a)$ and $N(r, a)$ in the invariant sum $m+N$. An answer was given by Nevanlinna with great accuracy in the form of an inequality which he called the second main theorem. While the problem leading to the second main theorem can be conveniently formulated with the first main theorem at hand, Nevanlinna actually arrived at the main theorems simultaneously. Both were announced for the first time in the Comptes Rendus note [19] of July 1924, a true landmark in the theory of meromorphic functions.

At the initial stage of his research Nevanlinna concentrated chiefly on questions emanating from Borel's theorem, being in no great hurry to deal with the general case of meromorphic functions. It is true that in the breakthrough paper [16] cited in Section 2.4, he established formula (7), i.e. almost proved the first main theorem, for meromorphic functions. But this paper was primarily devoted to the study of the growth of regular analytic functions when the distribution of $a$-points 
was known for two values of $a$. The Nevanlinna functions $m$ and $N$ were used for that purpose, $m(r, f)$ in place of the classical measure $\log M(r)$ and $N(r, a)$ instead of $n(r, a)$. Thus Nevanlinna was automatically led to compare the growth of $m(r, f)$ and $N(r, a)$, even before he had observed the invariance of $m+N$ and introduced the characteristic function.

The paper [16] already contained a preliminary version of the second main theorem for regular analytic functions, i.e. for entire functions and for functions holomorphic in a disc. (About a year later, this result was to elicit important comments from Littlewood and Collingwood, as will be explained in Section 3.3.) But Nevanlinna was quick to observe that the use of $m(r, f)$ instead of $\log M(r)$ made his method applicable to meromorphic functions as well. In the July 1924 Comptes Rendus note he was ready to announce the basic inequality for functions meromorphic in the plane, in the case of three given values:

$$
T(r)<N(r, a)+N(r, b)+N(r, c)+S(r),
$$

where $S(r)$ is in general small compared to $T(r)$.

This inequality shows that in the equation (9) of the first main theorem the term $N(r, a)$ is usually larger than $m(r, a)$. For instance, if $\lim \sup N(r, a) / T(r)=0$ for two values of $a$, then $\lim \sup N(r, a) / T(r)=1$ for all other values of $a$.

3.2. Survey of the results. Inequality (1) was what Nevanlinna had wished to prove. He had also made the very important remark in [17] that a natural definition for the order of a meromorphic function is the upper limit

$$
\limsup _{r \rightarrow \infty} \frac{\log T(r)}{\log r}
$$

in the case of entire functions $T(r)=m(r, \infty)$, and a simple estimation shows that this definition agrees with the classical definition (6) in Chapter 1.

All in all, Nevanlinna was now in a position to present a large survey of his theory of meromorphic functions. This he did in 1925 in the paper [20] "Zur Theorie der meromorphen Funktionen", which must be regarded as Nevanlinna's main work. In 1943, H. Weyl [35] wrote about it: "The appearance of this paper has been one of the few great mathematical events in our century."

A central position in [20] is occupied by the first and second main theorems, there so named for the first time. The proof of the second main theorem, extraordinary in its originality, can be followed in all details. Technically it is far too complicated to be reproduced here. A basic idea, to compare the growth of the function with that of its derivative, goes back to Borel [3]. The technical starting point is the omnipresent Poisson-Jensen formula (2) of Chapter 2. It first yields a representation for $\log f$, and then through differentiation, for $f^{\prime} \mid f$. This makes it possible to estimate $m\left(r, f^{\prime} \mid f\right)$, which is the key for estimating the remainder term $S(r)$. 
The other results of [20] were deduced more or less as direct consequences of the main theorems. The use of the characteristic $T(r)$ in the place of $\log M(r)$ enabled Nevanlinna to establish for meromorphic functions practically the whole classical theory of entire functions. Results on entire functions were thus obtained in a unified manner as special cases of more general theorems. More than that, Nevanlinna's main theorems often yielded these results in a sharper form or under weaker hypotheses. And finally, due to the improved results, new problems were opened.

For functions meromorphic in the unit disc, both main theorems hold in the same form as in the case of the plane. The interpretation of the first main theorem is of interest if the characteristic $T$ is unbounded. But the case of a bounded $T$ is not without interest either: Nevanlinna had had time to prove in [18], as a generalization of a previous result in [13], that the class of meromorphic functions of bounded characteristic coincides with the class of functions which are quotients of two bounded analytic functions.

In the second main theorem, $S(r)$ plays the role of the remainder term provided

$$
\limsup _{r \rightarrow 1} T(r) / \log \frac{1}{1-r}=\infty .
$$

This is the critical rate of growth: under this condition and only under it, do the conclusions drawn from the second main theorem for functions meromorphic in the plane remain valid.

3.3. Remarks of Littlewood and Collingwood. In spite of the richness of results and the superb style, without a supplement written slightly later, the paper [20] would not have given the main features of the theory in the best possible form. In building his theory, Nevanlinna concentrated on studying properties of a meromorphic function $f$ with the distribution of the roots $f(z)=a$ given for three different values of $a$. He once told me that at an early stage he felt that there was a version of Picard's theorem still sharper than Borel's improvement but that for quite a while he did not know exactly in which direction to pursue it. Inequality (1) was a most satisfactory solution, and this may explain why Nevanlinna failed to notice that his method actually applied to the case in which arbitrarily many values are given instead of just three.

This observation was made independently by Littlewood and Collingwood, after they had analyzed the proof of the preliminary inequality in Nevanlinna's paper [16]. Nevanlinna had completed the manuscript for [20], when he received a letter from Littlewood relating about this. Almost simultaneously, in November 1924, Collingwood made the same remark in the Comptes Rendus note [6]. This prompted Nevanlinna to write an appendix which could still be included in [20]. With the sole difference that $q(\geqq 3)$ values were considered rather than 3 , he reformulated the Second Main Theorem: 
Let $f$ be meromorphic in the complex plane and let $a_{1}, a_{2}, \ldots, a_{q}$ be distinct finite or infinite numbers. Then

$$
(q-2) T(r)<\sum_{i=1}^{q} N\left(r, a_{i}\right)-N_{1}(r)+S(r),
$$

where $N_{1}(r) \geqq 0$ and $S(r)$ is in general essentially smaller than $T(r)$.

More precisely,

$$
N_{1}(r)=\int_{0}^{r} \frac{n_{1}(t)}{t} d t,
$$

where $n_{1}(r)$ is the number of all multiple $a$-points of $f$ in $|z| \leqq r$, an $a$-point of multiplicity $k$ being counted $k-1$ times. The remainder term satisfies the condition $S(r)=O(\log (r T(r)))$, except perhaps for a set of values $r$ of finite length. If $f$ is of finite order, then $S(r)=O(\log r)$.

3.4. Deficiency relation. The general form (2) of the second main theorem proved to be of paramount importance: it permitted the value distribution theory to be pushed far beyond its classical scope. Nevanlinna immediately drew the first decisive conclusions in the appendix of [20]. From the inequality (2) it first follows that

$$
\limsup _{r \rightarrow \infty} \frac{N(r, a)}{T(r)}=1,
$$

with the possible exception of countably many values of $a$. Consequently, up to such exceptional values, a meromorphic function exhibits great symmetry in its value distribution, again resembling the behaviour of rational functions. The relation (3) generalized the result of Collingwood [6] that for entire functions of finite order the inequality $\lim \sup N(r, a) / m(r, f) \leqq \Theta<1$ is possible for at most $[1 /(1-\Theta)]$ different values of $a$.

To measure the deviation from normality Nevanlinna introduced the number

$$
\delta(a)=1-\limsup _{r \rightarrow \infty} N(r, a) / T(r) ;
$$

a few years later he began to call it the deficiency of $a$. Clearly $0 \leqq \delta(a) \leqq 1$, and if $f$ omits the value $a$, then $\delta(a)=1$. By the result (3), $\delta(a)>0$ for only countably many values of $a$.

Typically for Nevanlinna, once the right concept (4) was defined, obtaining a striking result was just a matter of observation. Inequality (2) yields immediately the Deficiency Relation:

The deficiencies of a meromorphic function satisfy the inequality

$$
\sum_{a} \delta(a) \leqq 2 .
$$

This is a far-reaching refinement of Picard's and Borel's theorems. 


\section{Riemann surfaces of meromorphic functions}

4.1. Inverse problem and multiple values. With the appearance of the paper [20], the birth of the Nevanlinna theory was complete and its full maturity was near at hand. However, the concept of deficiency and a closer analysis of the second main theorem with attention to the term $N_{1}$ generated completely new problems. They led to the study of the Riemann surfaces onto which meromorphic functions map the complex plane. This opened a new road into the theory of meromorphic functions which proved to be strongly differential-geometric in nature. Therefore, a few words about the early stages of this novel development can and perhaps even should be incorporated in an exposition about the birth of the Nevanlinna theory.

The new turn in the theory was not quite immediate. It is true that, as early as 1924, Collingwood [7] had analyzed the properties of the Riemann surface of an entire function of finite order over a point $a$ for which $\delta(a)>0$. It seems, however, that for a while this viewpoint did not attract great attention. With Nevanlinna the first hints of a new approach to meromorphic functions appear in the monograph [21], whose preface is dated November 1927, three years after the writing of [20] was completed. First, Nevanlinna there explicitly posed the Inverse Problem for the deficiency relation, in the following form:

Given the numbers $\delta_{v}, 0<\delta_{v} \leqq 1, \sum \delta_{v}=2$, find a meromorphic function $f$ which at prescribed points $a_{v}$ has the deficiencies $\delta\left(a_{v}\right)=\delta_{v}$.

In the second main theorem Nevanlinna turned his attention to the term $N_{1}(r)$ arising from multiple roots of the equation $f(z)=a$. If $n_{1}(r, a)$ denotes the number of multiple $a$-points in $|z| \leqq r$, an $a$-point of multiplicity $k$ being counted $k-1$ times, and $N_{1}(r, a)$ the corresponding integrated counting function, then $N_{1}(r)=$ $\sum_{a} N_{1}(r, a)$. In order to measure the relative frequency of multiple values, Nevanlinna introduced the number

$$
\theta(a)=\liminf _{r \rightarrow \infty} N_{1}(r, a) / T(r)
$$

It follows that $\theta(a) \geqq 0, \theta(a) \leqq 1-\delta(a)$, and $\theta(a)=0$, except perhaps for countable many values of $a$. The second main theorem yields immediately the inequality

$$
\sum \delta(a)+\sum \theta(a) \leqq 2
$$

This is a remarkable sharpening of the deficiency relation.

In [21], where (2) was stated and important conclusions drawn from it, Nevanlinna did not yet use geometric language. He called $\theta(a)$ the multiplicity index, but changed it soon to ramification index (Verzweigungsindex). 
4.2. Methodological remarks. Nevanlinna soon realized that in order to better understand the deficiency relation and to attack the inverse problem one should subject to closer scrutiny the Riemann surfaces of meromorphic functions. The ramification index is also directly connected with these surfaces, since $\theta(a)>0$ if the corresponding surface is sufficiently branched over the point $a$.

At this same time, other geometric aspects were brought into the value distribution theory. In $1927 \mathrm{~F}$. Nevanlinna had given a new proof for the second main theorem by studying the universal covering surface of the plane punctured at the points $a_{1}, a_{2}, \ldots, a_{q}, q \geqq 3$. This proof, a return to Picard's original idea, was very much to Rolf Nevanlinna's taste, as is confirmed by his many remarks later. And by 1929, Shimizu and Ahlfors, Nevanlinna's first student, had both shown that the Nevanlinna characteristic of a meromorphic function $f$ admits the representation

$$
T(r)=\int_{0}^{r} \frac{A(t)}{t} d t
$$

where $A(t)$ denotes the spherical area divided by $\pi$ of the part of the Riemann surface of $f$ which is the image of the disc $|z| \leqq t$.

It might be appropriate to briefly point out that besides Nevanlinna's standard method, the use of the harmonic function $\log |f|$ and the resulting Jensen's formula, his theory admits a second easy approach. Through it further light can be shed on the results of Shimizu-Ahlfors and F. Nevanlinna.

First of all, Jensen's formula can be readily obtained with the aid of the principle of argument

$$
\int_{|z|=r} d \arg (f(z)-a)=2 \pi(n(r, a)-n(r, \infty)) .
$$

By applying the Cauchy-Riemann equation $\partial \arg f / \partial \theta=r \partial \log |f| / \partial r$ and then integrating (4) with respect to $r$, one gets Jensen's formula, directly in the form

$$
\frac{1}{2 \pi} \int_{0}^{2 \pi} \log \left|f\left(r e^{i \theta}\right)-a\right| d \theta-\log |f(0)-a|=N(r, a)-N(r, \infty) .
$$

Let us now integrate (5) once more, with respect to a measure $\mu(a)$ with support $E$ and total mass 1 . With the notation

$$
u(w)=\int_{E} \log |w-a| d \mu(a)
$$

for the associated logarithmic potential, it follows from (5) that

$$
\frac{1}{2 \pi} \int_{0}^{2 \pi} u\left(f\left(r e^{i \theta}\right)\right) d \theta-u(f(0))=\int_{E} N(r, a) d \mu(a)-N(r, \infty) .
$$


If the order of integration is changed, the right-hand mean value of $N$ assumes the form

$$
\int_{E} N(r, a) d \mu(a)=\int_{0}^{r} \frac{\Omega(t)}{t} d t,
$$

where $\Omega(t)$ is the mass on the image of $|z| \leqq t$. (The general relation (6) was first derived by Frostman in 1934.)

If $E$ is the extended plane and $\mu$ the normalized spherical area, then by subtracting (5) from (6) one obtains Nevanlinna's first main theorem, with the characteristic in the Shimizu-Ahlfors form (3). The approach of F. Nevanlinna to the second main theorem amounts to comparing the characteristic, i.e. the mean value of $N$ with respect to the spherical area, to the mean value of $N$ with respect to the hyperbolic area of the plane punctured at the points $a_{1}, a_{2}, \ldots, a_{q}$.

4.3. Branching of Riemann surfaces. In Nevanlinna's paper [22], which is the lecture he gave in the 1929 Scandinavian Congress of Mathematicians in Oslo, Riemann surfaces of meromorphic functions are for the first time the main theme. In the paper [23] "Über die Herstellung transzendenter Funktionen als Grenzwerte rationaler Funktionen" of 1930, he started from the classical Riemann formula concerning the branch points of an $n$-sheeted planar Riemann surface. Suppose a meromorphic $f$ is approximated by rational functions $f_{n}$ of order $n$. If $\bar{n}(a)$ denotes the number of different roots of $f_{n}(z)=a$ in the extended plane, then $n-\bar{n}(a)$ is the sum of the orders of branch points over $a$ of the Riemann surface of $f_{n}$. By Riemann's formula, $\sum_{a}(n-\bar{n}(a))=2 n-2$, and consequently as $n \rightarrow \infty$,

$$
\sum_{a}(1-\bar{n}(a) / n)=2-2 / n \rightarrow 2
$$

Nevanlinna compared this relation with the formula $\sum \delta(a)+\sum \theta(a) \leqq 2$. For the rational function $f_{n}$, we have $\theta(a)=1-\bar{n}(a) / n$ and $\delta(a)=0$ for all values $a$. The emergence of deficient values of $f=\lim f_{n}$ was envisaged as follows. Assume that over $a$ the Riemann surface of $f_{n}$ has a branch point whose order divided by $n$ tends to a positive limit $\delta$ as $n \rightarrow \infty$. The corresponding preimage of $a$ then tends to $\infty$, and this means that a certain portion of the roots of $f(z)=a$ gets lost producing the deficiency $\delta$. "A deficient value is taken at $\infty$ so many times that it compensates for the deficiency in the finite plane", an analogue to the behaviour of rational functions.

Explaining this and similar heuristic ideas and testing them by means of examples formed an essential part of Nevanlinna's survey lectures [24] at Hamburg University in 1931.

The new geometric way of handling meromorphic functions soon produced concrete results. For instance, through an analysis of the class of Riemann surfaces with finitely many logarithmic branch points Nevanlinna gave in 1932 ([25]) 
the first partial solution of the inverse problem: finitely many rational deficiencies can be prescribed. (Only much later, in 1977, a complete solution was obtained when Drasin [8], generalizing Nevanlinna's method in [25] and using quasiconformal mappings, proved that both the deficiencies and the ramification indexes can be assigned arbitrarily. In this sense, inequality (2) tells the whole truth about the value distribution of meromorphic functions.)

4.4. New directions of research. The character of the value distribution theory was now clearly changing. In the preface of [24] Nevanlinna said that in the theory of meromorphic functions the ultimate goal must be a closer knowledge of their Riemann surfaces. He listed a number of concrete problems and, with geometric interpretations in mind, announced the desirability of getting results in terms of the original simple counting function $n(r, a)$ instead of its integrated mean $N(r, a)$. (This was soon to happen through the work of Ahlfors.)

With characteristic lucidity, Nevanlinna described the geometric view-point in the lecture [26] "Über die Riemannsche Fläche einer analytischen Funktion" in the 1932 International Congress of Mathematicians in Zürich. Having remarked that the distribution of $a$-points of a meromorphic function $f$ near an isolated singularity is very uniform for most values $a$, Nevanlinna declared the main problem of the value distribution theory to be the study of the exceptional values $a$, or equivalently, the study of the branching properties of the Riemann surface of $f$.

On the Riemann surface of $f$ the inverse $f^{-1}$ is a single-valued univalent analytic function. Thus a connection was established between the theory of such functions and the value distribution theory. The "type problem", i.e. to decide whether a simply connected Riemann surface is of hyperbolic or parabolic type, found direct contact with the Nevanlinna theory.

The study of Riemann surfaces advocated by Nevanlinna led to vast new research, partly of topological nature, which was succesfully carried out by Ahlfors and others. A survey of the developments until the mid-thirties is presented in Nevanlinna's monograph [27]. Still today the Nevanlinna theory even in its purest classical form is very much alive, and it has led to generalizations far beyond the theory of functions meromorphic in the complex plane.

\section{References}

[1] Ahlfors, L.: Das mathematische Schaffen Rolf Nevanlinnas. - Ann. Acad. Sci. Fenn. Ser A I Math. 2, 1976, 1-15.

[2] Blumenthal, O.: Principes de la théorie des fonctions entières d'ordre infini. - Collection de monographies sur la théorie des fonctions, Gauthier-Villars, Paris, 1910.

[3] Borel, É.: Sur les zéros des fonctions entières. - Acta Math. 20, 1897, 357-396.

[4] BoRel, É.: Leçons sur les fonctions entières. Deuxième Edition revue et augmentée d'une Note de M. G. Valiron. - Collection de monographies sur la théorie des fonctions. Gauthier-Villars et $\mathrm{C}^{\mathrm{1} \text { e }}$, Paris, 1921. 
[5] Borel, É.: Leçons sur les fonctions méromorphes. - Collection de monographies sur la théorie des fonctions, Gauthier-Villars, Paris, 1903.

[6] Collingwood, E. F.: Sur quelques théorèmes de M. Nevanlinna. - C. R. Acad. Sci. Paris 179, 1924, 955-957.

[7] Collingwood, E. F.: Sur les valeurs exceptionnelles des fonctions entières d'ordre fini. - C. R. Acad. Sci. Paris 179, 1924, 1125-1128.

[8] Drasin, D.: The inverse problem of the Nevanlinna theory. - Acta Math. 138, 1977, 83-151.

[9] Hadamard, J.: Étude sur les propriétés des fonctions entières et en particulier d'une fonction considerée par Riemann. - J. Math. Pures Appl. 9, 1893, 171-215.

[10] Hayman, W. K.: Meromorphic functions. - Clarendon Press, Oxford, 1964.

[11] Jensen, J. L. W. V.: Sur un nouvel et important théorème de la théorie des fonctions. - Acta Math. 22, 1899, 359-364.

[12] LindelöF, E.: Mémoire sur la théorie des fonctions entières de genre fini. - Acta Soc. Sci. Fenn. $31: 1,1902,1-79$.

[13] Nevanlinna, F. und R.: Über die Eigenschaften analytischer Funktionen in der Umgebung einer singulären Stelle oder Linie. - Acta Soc. Sci. Fenn. 50:5, 1922, 1-46.

[14] Nevanlinna, R.: Sur les relations qui existent entre l'ordre de croissance d'une fonction monogène et la densité de ses zéros. - C. R. Acad. Sci. Paris 174, 1922, 1325-1327.

[15] Nevanlinna, R.: Über die Anwendung des Poisson'schen Integrals zur Untersuchung der Singularitäten analytischer Funktionen. - Matematikerkongressen i Helsingfors den 4-7 juli 1922, Akademiska Bokhandeln, Helsingfors 1923, 273-289.

[16] Nevanlinna, R.: Untersuchungen über den Picard'schen Satz. - Acta Soc. Sci. Fenn. 50:6, 1924, $1-42$.

[17] Nevanlinna, R.: Sur les fonctions méromorphes. - C. R. Acad. Sci. Paris 178, 1924, 367_. 370.

[18] Nevanlinna, R.: Über eine Klasse meromorpher Funktionen. - Math. Ann. 92, 1924, 145154.

[19] Nevanlinna, R.: Sur les valeurs exceptionnelles des fonctions méromorphes. - C.R. Acad. Sci. Paris 179, 1924, 24-26.

[20] Nevanlinna, R.: Zur Theorie der meromorphen Funktionen. - Acta Math. 46, 1925, 1-99.

[21] Nevanlinna, R.: Le théorème de Picard-Borel et la théorie des fonctions méromorphes. Collection de monographies sur la théorie des fonctions, Gauthier-Villars et $\mathrm{C}^{\mathrm{i} \text {, }}$, Paris, 1929.

[22] Nevanlinna, R.: Über gewisse neuere Ergebnisse in der Theorie der Wertverteilung. - Den syvende skandinaviske Matematikerkongress i Oslo 19-22 august 1929, A. W. Brøggers boktrykkeri a/s, Oslo, 1930, 68-80.

[23] Nevanlinna, R.: Über die Herstellung transzendenter Funktionen als Grenzwerte rationaler Funktionen. - Acta Math. 55, 1930, 259-276.

[24] Nevanlinna, R.: Über die Werteverteilung der eindeutigen analytischen Funktionen. - Abh. Math. Sem. der Hamburgischen Univ. 8:4, 1931, 351-400.

[25] Nevanlinna, R.: Über Riemannsche Flächen mit endlich vielen Windungspunkten. - Acta Math. 58, 1932, 295-373.

[26] Nevanlinna, R.: Über die Riemannsche Fläche einer analytischen Funktion. - Verh. des Internationalen Mathematiker-Kongresses Zürich 1932, 1. Band, Orell Füssli Verlag, Zürich u. Leipzig, 221-239.

[27] Nevanlinna, R.: Eindeutige analytische Funktionen. - Die Grundlehren der mathematischen Wissenschaften 46, Verlag von Julius Springer, Berlin, 1936.

[28] Nevanlinna, R.: Muisteltua. - Otava, Helsinki, 1976.

[29] PiCARD, É.: Sur une propriété des fonctions entières. - C. R. Acad. Sci. Paris 88, 1879, 1024-1027. 
[30] Poincaré, H.: Sur les fonctions entières. - Bull. Soc. Math. France 11, 1883, 136-144.

[31] Valiron, G.: Sur les fonctions entières d'ordre fini et d'ordre nul et en particulier les fonctions à correspondance régulière. - Thése, Ann. Fac. Toulouse, 1913.

[32] Valiron, G.: Sur la théorie des fonctions entières. - C. R. Congrès International des Mathématiciens, Strasbourg 22-30 Septembre 1920, Imprimerie et Librairie Edouard Privat, Toulouse, 1921.

[33] VAliRon, G.: Lectures on the general theory of integral functions. - Imprimerie et Librairie Edouard Privat, Toulouse, 1923.

[34] Weierstrass, K.: Zur Theorie der eindeutigen analytischen Funktionen. - Abh. Königl. Akad. Wiss. Berlin 1876, 11-60, reprinted Math. Werke, Band II, Berlin, 1895, 77-124.

[35] WeYL, H. (in collaboration with F. J. WeYL): Meromorphic functions and analytic curves. Princeton University Press, Princeton, 1943.

University of Helsinki

Department of Mathematics

SF-00100 Helsinki 10

Finland

Received 5 March 1981 